\title{
Seasonal Variations in the Performance of Bivoltine Mulberry Silkworm (Bombyx mori L.) Breeds under Kashmir Climatic Conditions
}

\author{
Bharath Kumar Neelaboina ${ }^{1^{*}}$, Shivkumar ${ }^{2}$, Mir Nisar Ahmad ${ }^{3}$, Kiran $\mathrm{R}^{4}$, S. Roy Chowdhury ${ }^{5}$ \\ ${ }^{1}$ Scientist C, Silkworm Division, Central Sericultural Research and Training Institute, Pampore (J\&K), India \\ ${ }^{2}$ Scientist C, Silkworm Division, Central Sericultural Research and Training Institute, Pampore (J\&K), India \\ ${ }^{3}$ Scientist D, Silkworm Division, Central Sericultural Research and Training Institute, Pampore (J\&K), India \\ ${ }^{4}$ Scientist B, Silkworm Division, Central Sericultural Research and Training Institute, Pampore (J\&K), India \\ ${ }^{5}$ Director, Central Sericultural Research and Training Institute (CSR\&TI), Pampore, Jammu \& Kashmir, India
}

\begin{abstract}
*Address for Correspondence: Dr. Bharath Kumar Neelaboina, Scientist C, Silkworm Division, Central Sericultural Research and Training Institute (CSR\&TI), Central Silk Board, Gallandar, Pampore-192121, Jammu \& Kashmir, India E-mail: bharath.agrico@gmail.com
\end{abstract}

Received: 13 May 2020/ Revised: 15 Aug 2020/ Accepted: 24 Oct 2020

\begin{abstract}
Background: The present research was carried out to evaluate the performance of bivoltine silkworm, Bombyx mori L., breeds (CSR2, CSR6, CSR27, CSR26, CSR50, PAM114, PAM117, APS4, APS5, SK6 and SK7) of different origin under Kashmir climatic conditions during spring (May-June), summer (July-August) and autumn (August-September), 2019 at Central Sericultural Research and Training Institute, Pampore, Jammu and Kashmir, India.

Methods: These eleven silkworm parental breeds were evaluated for their performance in nine metric traits viz., fecundity (No), hatching (\%) yield per 10,000 larvae by number and by weight ( $\mathrm{kg})$, single cocoon weight $(\mathrm{g})$, single shell weight (g), shell ratio (\%), pupation rate $(\%)$ and Filament length $(\mathrm{m})$. The data generated were analyzed statistically and subjected to multiple trait Evaluation Index (E.I). The rearing was carried out under the uniformed laboratory condition by adopting the standard method.

Results: Based on the evaluation index values ranking CSR2, CSR26, CSR27, PAM114 and PAM117 performed well in all the three seasons with E.I value above 50. APS4 has performed well in spring and summer season whereas CSR50 recorded E.I value above 50 in summer and autumn only. Three breeds viz., CSR6, APS5 and SK7 recorded E.I value below 50 in all the three seasons. SK6 recorded E.I value above 50 only in the spring season.

Conclusion: The occurrence of seasonal variation in the performance is due to the silkworm breeds, which originated from different progenitors coupled with the change in climatic conditions during three seasons (spring, summer and autumn) in Kashmir valley.
\end{abstract}

Key-words: Evaluation index, Mulberry Silkworm, Seasonal variation, Silkworm breeds, Sericulture

\section{INTRODUCTION}

The mulberry silkworm B. mori L. (Lepidoptera: Bombycidae) domesticated for silk production is the most well-studied lepidopteran model system because of its rich repertoire of well-characterized mutations affecting virtually every aspect of the organism's

\section{How to cite this article}

Neelaboina BK, Kumar S, Ahmad MN, Kiran R, Chowdhury SR. Seasonal Variations in the Performance of Bivoltine Mulberry Silkworm (Bombyx mori L.) Breeds under Kashmir Climatic Conditions. SSR Inst. Int. J. Life Sci., 2020; 6(6): 2678-2686.

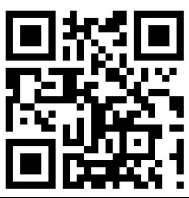

morphology, development, behaviour and its considerable importance ${ }^{[1]}$. The commercial silkworm rearing in Kashmir region can be conducted during spring season (May-June) summer season (July- August) and autumn season (August- September). But the majority of sericulture activities are being carried out during spring season which is congenial for silkworm rearing. So far only few silkworm breeds and hybrids were developed specifically for the spring season in Jammu and Kashmir ${ }^{[2-4]}$. In the case of summer season only $10-15 \%$ of farmers take up sericulture activities ${ }^{[5]}$ and attempts were made for the development and identification of hybrids for autumn and summer season ${ }^{[6-10]}$. 
Despite all the efforts, CSR double hybrid developed in the southern part of the country is commercially popular in this region among sericulture rearers.

Selection of parental material is the crucial step to develop hybrids suitable for Kashmir climatic conditions for which systematic breeding approach by silkworm breeders will play an important role ${ }^{[11-13]}$. To select the parental stocks, one should have thorough knowledge regarding the diversity in the genetic constitution along with the traits that need to be improved in the hybrid material. Genetic diversity in the parental material is directly proportional to the chances of obtaining a higher amount of heterotic potential and also more variability is expected to appear in segregating generations of such crosses $^{[14]}$. The impact of the exploitation of heterosis is seen in the sericulture industry in terms of growth, vigour and economic traits of silkworm hybrids compared to pure breeds.

The crossing of genetically diverse parents helps in the recombination of genes from diverse resources [15] producing high heterotic effects and more variability in segregating generations.
Hence selection of genetically pure and divergent parental strains is critical to the success of a hybridization programme in silkworm ${ }^{[1]}$. It is also needed for the hour to develop silkworm hybrids/ double hybrids withstanding climate change ${ }^{[16]}$.

Hence, the present study aims to evaluate the performance of breeds in all the three seasons at CSR\&TI, Pampore to develop sustainable bivoltine silkworm double hybrids suitable for Kashmir valley.

\section{MATERIALS AND METHODS}

The present investigation was carried out at Central Sericultural Research and Training Institute, Pampore, Jammu \& Kashmir (J\&K) during spring (May-June), summer (July-August) and autumn (August- September), 2019 to study and assess the seasonal variation in the performance of eleven silkworm breeds (CSR2, CSR6, CSR27, CSR26, CSR50, PAM114, PAM117, APS4, APS5, SK6 and SK7) of different origin under Kashmir climatic conditions. The characteristics of the parental breeds are presented in Table 1.

Table 1: Characteristic features of the eleven silkworm parental breeds

\begin{tabular}{ccccc}
\hline S.No. & Breeds & Larval Marking & Cocoon Colour & Cocoon shape \\
\hline 1 & CSR2 & Plain & White & Oval \\
2 & CSR6 & Marked & White & Constricted \\
3 & CSR26 & Marked & White & Constricted \\
4 & CSR27 & Plain & White & Oval \\
5 & CSR50 & Plain & White & Oval \\
6 & PAM114 & Plain & White & Oval \\
7 & PAM117 & Plain & White & Constricted \\
8 & APS4 & Plain & White & Constricted \\
9 & APS5 & Plain & White & Oval \\
10 & SK6 & Plain & White & Constricted \\
11 & SK7 & Plain & White & Constricted \\
\hline
\end{tabular}

The important quantitative and qualitative traits viz., fecundity, hatching percentage, yield per 10,000 larvae by weight, single cocoon weight, single shell weight, shell ratio, pupation rate and Filament length were recorded in all the eleven silkworm breeds during spring, summer and autumn, 2019. All the breeds were reared following completely randomised design with three replications each and 250 larvae were maintained in each replication after the $3^{\text {rd }}$ moult.
At the end of $5^{\text {th }}$ instar, the spinning larvae were collected manually and mounted in plastic collapsible mountages. The standard rearing techniques were followed. The evaluation index value was calculated for all the nine traits studied. The evaluation index (EI) was calculated as per the below-mentioned procedure ${ }^{[17]}$. 


\section{Evaluation Index $=\frac{A-B}{C} \times 10+50$}

Where, $A=$ Value obtained for a particular trait in a particular breed

$B=$ Mean value of a particular trait of all the breeds

$\mathrm{C}=$ Standard deviation of a particular trait of all the breeds

$10=$ Standard unit

$50=$ Fixed value

The index value obtained for all the traits was combined and the average El values were obtained. The El value fixed for the selection of a breed was 50 or $>50$. The line, which scored above the limit, is considered to possess greater economic value.

\section{RESULTS}

The perusal of spring 2019 parental data presented in Table 2 revealed that highest fecundity (568), hatching percentage (96.08), yield/10,000 larvae by weight (15.76), single cocoon weight (1.78), shell ratio (21.14) and filament length (921) observed in SK6, SK6, CSR2, PAM117, CSR27 and CSR2 respectively. Average multitraits evaluation Index of spring 2019 parental data

presented in Table 3 and same was depicted as a graph in Fig. 1 revealed that Seven breeds viz., CSR2 (54.29), CSR26 (52.90), CSR27 (56.74), PAM114 (51.85), PAM117 (57.51), APS4 (51.17) and SK6 (50.00) were performed well in the spring season except for CSR50 (42.90), SK7 (46.39), CSR6 (42.77) and APS5 (43.57).

Table 2: Rearing performance of the eleven silkworm parental breeds during spring, 2019

\begin{tabular}{|c|c|c|c|c|c|c|c|c|c|c|}
\hline \multirow{2}{*}{ S.No. } & \multirow{2}{*}{$\begin{array}{c}\text { Parental } \\
\text { breeds }\end{array}$} & \multirow{2}{*}{$\begin{array}{c}\text { Fecundi } \\
\text { ty } \\
\text { (No.) }\end{array}$} & \multirow{2}{*}{$\begin{array}{c}\text { Hatching } \\
(\%)\end{array}$} & \multicolumn{2}{|c|}{$\begin{array}{l}\text { Yield / 10, } 000 \\
\text { larvae brushed }\end{array}$} & \multirow{2}{*}{$\begin{array}{c}\text { Single } \\
\text { cocoon } \\
\text { wt. (g) }\end{array}$} & \multirow{2}{*}{$\begin{array}{c}\text { Single } \\
\text { shell } \\
\text { wt. } \\
\text { (g) }\end{array}$} & \multirow{2}{*}{$\begin{array}{c}\text { Shell } \\
\text { ratio } \\
(\%)\end{array}$} & \multirow{2}{*}{$\begin{array}{c}\text { Pupatio } \\
\text { n rate } \\
\text { (\%) }\end{array}$} & \multirow{2}{*}{$\begin{array}{c}\text { Filament } \\
\text { length }(\mathrm{m})\end{array}$} \\
\hline & & & & By No. & $\begin{array}{l}\text { By Wt. } \\
\text { (kg) }\end{array}$ & & & & & \\
\hline 1 & CSR-2 & 484 & 92.52 & 9700 & 15.76 & 1.74 & 0.37 & 21.08 & 93.00 & 921 \\
\hline 2 & CSR-6 & 437 & 94.52 & 9387 & 12.97 & 1.54 & 0.32 & 20.87 & 90.00 & 754 \\
\hline 3 & CSR-26 & 552 & 95.30 & 9640 & 15.61 & 1.70 & 0.35 & 20.69 & 93.00 & 700 \\
\hline 4 & CSR-27 & 567 & 95.32 & 9667 & 15.42 & 1.72 & 0.36 & 21.14 & 93.00 & 816 \\
\hline 5 & CSR-50 & 515 & 94.50 & 9040 & 13.34 & 1.58 & 0.32 & 20.31 & 87.00 & 800 \\
\hline 6 & Pam-114 & 467 & 94.80 & 9667 & 15.03 & 1.70 & 0.34 & 20.14 & 94.00 & 823 \\
\hline 7 & Pam-117 & 563 & 95.40 & 9707 & 15.61 & 1.78 & 0.37 & 20.53 & 94.00 & 814 \\
\hline 8 & APS-4 & 532 & 94.50 & 9760 & 15.64 & 1.60 & 0.32 & 19.65 & 94.00 & 816 \\
\hline 9 & APS-5 & 553 & 95.50 & 9060 & 14.02 & 1.55 & 0.30 & 19.33 & 87.00 & 796 \\
\hline 10 & SK-6 & 568 & 96.08 & 9760 & 14.76 & 1.60 & 0.29 & 18.11 & 93.00 & 814 \\
\hline \multirow[t]{3}{*}{11} & SK-7 & 566 & 95.33 & 9640 & 12.76 & 1.47 & 0.28 & 19.00 & 93.00 & 845 \\
\hline & Avg. & 528 & 94.89 & 9548 & 14.63 & 1.64 & 0.33 & 20.08 & 91.91 & 809 \\
\hline & SD & 46 & 0.93 & 266 & 1.15 & 0.10 & 0.03 & 0.96 & 2.66 & 54 \\
\hline
\end{tabular}

Table 3: Evaluation of index values of eleven silkworm parental breeds during spring, 2019

\begin{tabular}{|c|c|c|c|c|c|c|c|c|c|c|c|}
\hline \multirow{2}{*}{ S.No. } & \multirow{2}{*}{$\begin{array}{c}\text { Parental } \\
\text { breeds }\end{array}$} & \multirow{2}{*}{$\begin{array}{c}\text { Fecun } \\
\text { dity } \\
\text { (No) }\end{array}$} & \multirow{2}{*}{$\begin{array}{c}\text { Hatchi } \\
\text { ng } \\
(\%)\end{array}$} & \multicolumn{2}{|c|}{$\begin{array}{l}\text { Yield / 10, } 000 \\
\text { larvae brushed }\end{array}$} & \multirow{2}{*}{$\begin{array}{l}\text { Single } \\
\text { cocoon } \\
\text { wt. (g) }\end{array}$} & \multirow{2}{*}{$\begin{array}{c}\text { Single } \\
\text { shell } \\
\text { wt. (g) }\end{array}$} & \multirow{2}{*}{$\begin{array}{l}\text { Shell } \\
\text { ratio } \\
(\%)\end{array}$} & \multirow{2}{*}{$\begin{array}{c}\text { Pupatio } \\
\text { n rate } \\
(\%)\end{array}$} & \multirow{2}{*}{$\begin{array}{c}\text { Filam } \\
\text { ent } \\
\text { length } \\
\text { (m) }\end{array}$} & \multirow{2}{*}{$\begin{array}{c}\text { Mean } \\
\text { E.I }\end{array}$} \\
\hline & & & & By No. & $\begin{array}{l}\text { By Wt. } \\
\text { (kg) }\end{array}$ & & & & & & \\
\hline 1 & CSR-2 & 40.46 & 24.55 & 55.73 & 59.81 & 60.73 & 62.16 & 60.43 & 54.10 & 70.68 & 54.29 \\
\hline 2 & CSR-6 & 30.35 & 46.02 & 43.93 & 35.60 & 40.35 & 47.71 & 58.28 & 42.83 & 39.85 & 42.77 \\
\hline 3 & CSR-26 & 55.35 & 54.42 & 53.47 & 58.53 & 56.62 & 57.34 & 56.41 & 54.10 & 29.88 & 52.90 \\
\hline 4 & CSR-27 & 58.55 & 54.65 & 54.47 & 56.86 & 58.55 & 61.09 & 61.13 & 54.10 & 51.29 & 56.74 \\
\hline
\end{tabular}


Cross ref DOI: 10.21276/SSR-IIJLS.2020.6.6.2

\begin{tabular}{cccccccccccc}
\hline 5 & CSR-50 & 47.17 & 45.84 & 30.88 & 38.83 & 43.89 & 47.18 & 52.41 & 31.56 & 48.34 & 42.90 \\
6 & Pam-114 & 36.76 & 49.07 & 54.47 & 53.45 & 57.13 & 54.67 & 50.67 & 57.85 & 52.58 & 51.85 \\
7 & Pam-117 & 57.81 & 55.51 & 55.98 & 58.48 & 64.67 & 61.62 & 54.71 & 57.85 & 50.92 & 57.51 \\
8 & APS-4 & 50.96 & 45.84 & 57.99 & 58.77 & 46.69 & 45.57 & 45.55 & 57.85 & 51.29 & 51.17 \\
9 & APS-5 & 55.58 & 56.58 & 31.63 & 44.73 & 41.44 & 40.76 & 42.22 & 31.56 & 47.60 & 43.57 \\
10 & SK-6 & 58.71 & 62.81 & 57.99 & 51.14 & 46.53 & 37.55 & 29.45 & 54.10 & 50.92 & 50.00 \\
11 & SK-7 & 58.30 & 54.70 & 53.47 & 33.81 & 33.42 & 34.34 & 38.74 & 54.10 & 56.65 & 46.39 \\
\hline
\end{tabular}

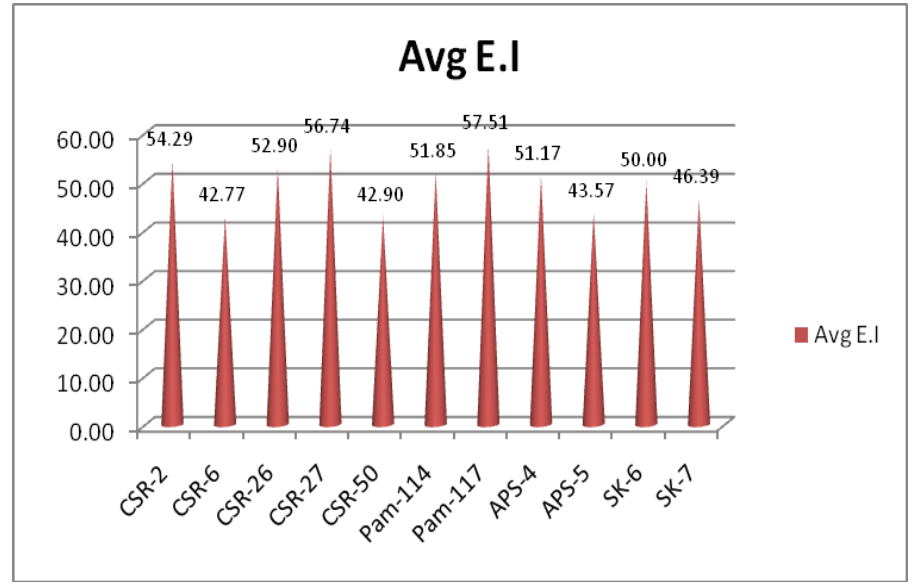

Fig. 1: Average multi-traits evaluation Index values of parental breeds during spring 2019

Further, summer 2019 data presented in Table 4 revealed that highest fecundity (546), hatching percentage $(95.77)$, yield $/ 10,000$ larvae by number (9240), yield/10,000 larvae by weight (14.72), single cocoon weight (1.67), shell ratio (20.30), pupation rate (88.00) and filament length (927) observed in CSR27, CSR2, PAM117, APS4, PAM117, CSR2, PAM117 and
CSR26 respectively. Average multi-traits evaluation Index of summer 2019 parental data was presented in Table 5 \& same was depicted as a graph in Fig. 2 revealed that, except CSR6 (41.44), APS5 (47.12), SK6 (42.02) and SK7 (43.88) remaining breeds viz., CSR2 (54.24), CSR26 (52.96), CSR27 (53.93), CSR50 (50.00), PAM114 (51.86), PAM117 (60.65) and APS4 (51.89) shown E.I above 50.

Table 4: Rearing performance of the eleven silkworm parental breeds during summer, 2019

\begin{tabular}{|c|c|c|c|c|c|c|c|c|c|c|}
\hline \multirow{2}{*}{ S.No. } & \multirow{2}{*}{$\begin{array}{c}\text { Parental } \\
\text { breeds }\end{array}$} & \multirow{2}{*}{$\begin{array}{c}\text { Fecun } \\
\text { dity } \\
\text { (No.) }\end{array}$} & \multirow{2}{*}{$\begin{array}{c}\text { Hatchi } \\
\text { ng } \\
(\%)\end{array}$} & \multicolumn{2}{|c|}{$\begin{array}{l}\text { Yield / 10, } 000 \\
\text { larvae brushed }\end{array}$} & \multirow{2}{*}{$\begin{array}{l}\text { Single } \\
\text { cocoon } \\
\text { wt. (g) }\end{array}$} & \multirow{2}{*}{$\begin{array}{c}\text { Single } \\
\text { shell wt. } \\
\text { (g) }\end{array}$} & \multirow{2}{*}{$\begin{array}{c}\text { Shell ratio } \\
\text { (\%) }\end{array}$} & \multirow{2}{*}{$\begin{array}{l}\text { Pupation } \\
\text { rate } \\
(\%)\end{array}$} & \multirow{2}{*}{$\begin{array}{c}\text { Filamen } \\
t \text { length } \\
\text { (m) }\end{array}$} \\
\hline & & & & By No. & $\begin{array}{l}\text { By Wt. } \\
\text { (kg) }\end{array}$ & & & & & \\
\hline 1 & CSR-2 & 542 & 95.77 & 8840 & 13.70 & 1.65 & 0.34 & 20.30 & 84.00 & 821 \\
\hline 2 & CSR-6 & 483 & 94.61 & 8507 & 12.52 & 1.63 & 0.32 & 19.81 & 83.00 & 830 \\
\hline 3 & CSR-26 & 510 & 94.97 & 8933 & 13.84 & 1.63 & 0.33 & 19.99 & 85.00 & 927 \\
\hline 4 & CSR-27 & 546 & 94.92 & 9000 & 13.17 & 1.63 & 0.33 & 20.21 & 86.00 & 871 \\
\hline 5 & CSR-50 & 543 & 94.77 & 8733 & 13.43 & 1.64 & 0.32 & 19.53 & 84.00 & 885 \\
\hline 6 & Pam-114 & 539 & 94.84 & 9160 & 13.23 & 1.59 & 0.31 & 19.24 & 87.00 & 906 \\
\hline 7 & Pam-117 & 541 & 95.03 & 9240 & 13.87 & 1.67 & 0.34 & 20.14 & 88.00 & 926 \\
\hline 8 & APS-4 & 529 & 94.05 & 9120 & 14.72 & 1.62 & 0.31 & 19.19 & 87.00 & 857 \\
\hline 9 & APS-5 & 537 & 94.83 & 9060 & 13.90 & 1.54 & 0.30 & 19.22 & 84.00 & 871 \\
\hline 10 & SK-6 & 528 & 94.23 & 9080 & 13.48 & 1.58 & 0.29 & 18.07 & 85.00 & 774 \\
\hline \multirow[t]{3}{*}{11} & SK-7 & 534 & 94.19 & 9000 & 13.50 & 1.61 & 0.29 & 18.01 & 85.00 & 809 \\
\hline & Avg. & 530 & 94.75 & 8970 & 13.58 & 1.62 & 0.31 & 19.43 & 85.27 & 861 \\
\hline & SD & 19 & 0.48 & 210 & 0.55 & 0.04 & 0.02 & 0.80 & 1.56 & 49 \\
\hline
\end{tabular}


Table 5: Evaluation index values of eleven silkworm parental breeds during summer, 2019

\begin{tabular}{|c|c|c|c|c|c|c|c|c|c|c|c|}
\hline \multirow[t]{2}{*}{ S.No. } & \multirow[t]{2}{*}{$\begin{array}{c}\text { Parental } \\
\text { breeds }\end{array}$} & \multirow{2}{*}{$\begin{array}{l}\text { Fecun } \\
\text { dity } \\
\text { (No.) }\end{array}$} & \multirow{2}{*}{$\begin{array}{c}\text { Hatchi } \\
\text { ng } \\
(\%)\end{array}$} & \multicolumn{2}{|c|}{$\begin{array}{l}\text { Yield / 10, } 000 \\
\text { larvae brushed }\end{array}$} & \multirow{2}{*}{$\begin{array}{l}\text { Single } \\
\text { cocoon } \\
\text { wt. (g) }\end{array}$} & \multirow{2}{*}{$\begin{array}{c}\text { Single } \\
\text { shell } \\
\text { wt. (g) }\end{array}$} & \multirow{2}{*}{$\begin{array}{l}\text { Shell } \\
\text { ratio } \\
(\%)\end{array}$} & \multirow{2}{*}{$\begin{array}{l}\text { Pupati } \\
\text { on } \\
\text { rate } \\
\text { (\%) }\end{array}$} & \multirow{2}{*}{$\begin{array}{l}\text { Filame } \\
\text { nt } \\
\text { length } \\
\text { (m) }\end{array}$} & \multirow[t]{2}{*}{$\begin{array}{c}\text { Mean } \\
\text { E.I }\end{array}$} \\
\hline & & & & By No. & $\begin{array}{l}\text { By Wt. } \\
\text { (kg) }\end{array}$ & & & & & & \\
\hline 1 & CSR-2 & 56.35 & 71.30 & 43.81 & 52.23 & 58.68 & 61.27 & 60.97 & 41.82 & 41.75 & 54.24 \\
\hline 2 & CSR-6 & 24.59 & 47.11 & 27.96 & 30.75 & 53.97 & 54.89 & 54.77 & 35.38 & 43.57 & 41.44 \\
\hline 3 & CSR-26 & 39.14 & 54.73 & 48.24 & 54.78 & 54.42 & 56.71 & 57.07 & 48.25 & 63.30 & 52.96 \\
\hline 4 & CSR-27 & 58.51 & 53.69 & 51.41 & 42.64 & 54.10 & 58.54 & 59.82 & 54.68 & 51.96 & 53.93 \\
\hline 5 & CSR-50 & 56.96 & 50.50 & 38.73 & 47.25 & 55.59 & 53.07 & 51.29 & 41.82 & 54.76 & 50.00 \\
\hline 6 & Pam-114 & 54.73 & 52.03 & 59.02 & 43.61 & 43.77 & 45.77 & 47.63 & 61.11 & 59.05 & 51.86 \\
\hline 7 & Pam-117 & 55.81 & 55.84 & 62.82 & 55.26 & 64.44 & 62.19 & 58.92 & 67.54 & 63.06 & 60.65 \\
\hline 8 & APS-4 & 49.40 & 35.43 & 57.12 & 70.79 & 49.42 & 47.60 & 47.05 & 61.11 & 49.07 & 51.89 \\
\hline 9 & APS-5 & 53.66 & 51.65 & 54.26 & 55.87 & 28.17 & 39.39 & 47.33 & 41.82 & 51.96 & 47.12 \\
\hline 10 & SK-6 & 48.81 & 39.28 & 55.22 & 48.22 & 39.34 & 33.92 & 32.94 & 48.25 & 32.23 & 42.02 \\
\hline 11 & SK-7 & 52.04 & 38.44 & 51.41 & 48.59 & 48.09 & 36.65 & 32.20 & 48.25 & 39.29 & 43.88 \\
\hline
\end{tabular}

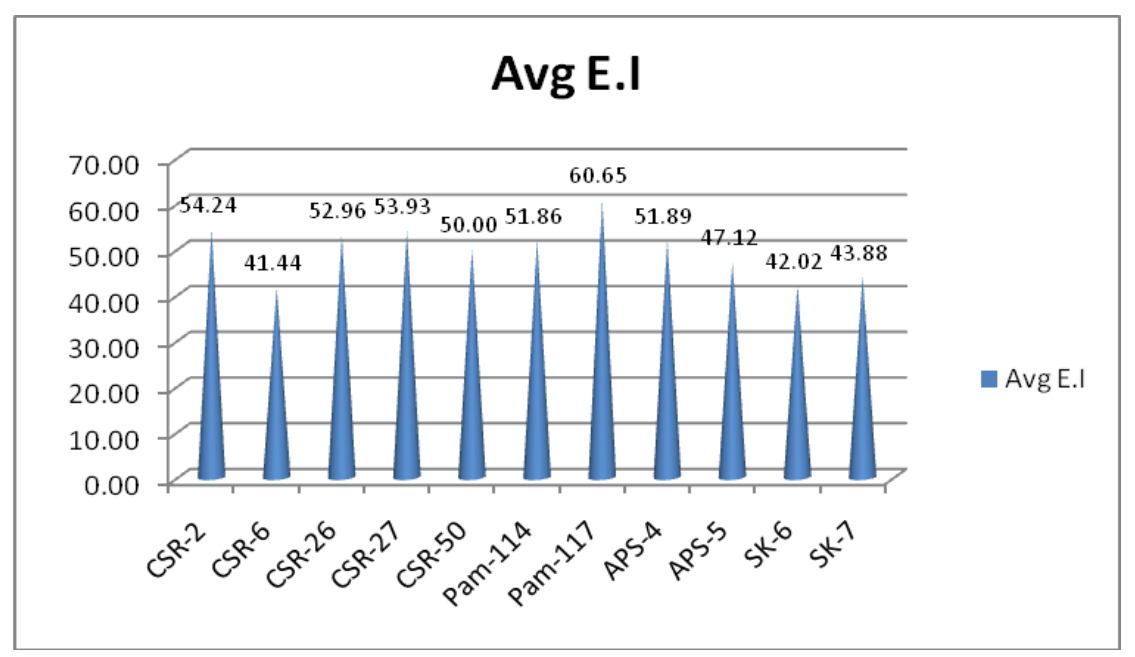

Fig. 2: Average multi-traits evaluation Index values of parental breeds during summer 2019

In case of autumn 2019, data presented in Table 6 revealed that highest fecundity (526), hatching percentage $(95.24)$, yield/10,000 larvae by number (9700), yield/10,000 larvae by weight (14.94), single cocoon weight (1.69), single shell weight $(0.35)$, shell ratio (20.73), pupation rate (94.50) and filament length (860) observed in SK6, CSR50, CSR2, CSR2, PAM114, PAM114, CSR2, CSR50 and PAM114 respectively.
Average multi-traits evaluation Index of autumn 2019 parental data was presented in Table $7 \&$ same was depicted as a graph in Fig. 3 revealed that, except CSR6 (36.82), APS4 (47.90), APS5 (45.53), SK6 (44.58) and SK7 (48.76) remaining breeds viz., CSR2 (54.69), CSR26 (52.68), CSR27 (52.73), CSR50 (52.95), PAM114 (60.00) and PAM117 (53.37) shown E.I above 50. 
Table 6: Rearing performance of the eleven silkworm parental breeds during autumn, 2019

\begin{tabular}{|c|c|c|c|c|c|c|c|c|c|c|}
\hline \multirow{2}{*}{ S.No. } & \multirow{2}{*}{$\begin{array}{l}\text { Parental } \\
\text { breeds }\end{array}$} & \multirow{2}{*}{$\begin{array}{c}\text { Fecun } \\
\text { dity } \\
\text { (No.) }\end{array}$} & \multirow{2}{*}{$\begin{array}{c}\text { Hatchin } \\
\text { g } \\
(\%)\end{array}$} & \multicolumn{2}{|c|}{$\begin{array}{l}\text { Yield / 10, } 000 \\
\text { larvae brushed }\end{array}$} & \multirow{2}{*}{$\begin{array}{l}\text { Single } \\
\text { cocoon } \\
\text { wt. (g) }\end{array}$} & \multirow{2}{*}{$\begin{array}{c}\text { Single } \\
\text { shell } \\
\text { wt. (g) }\end{array}$} & \multirow{2}{*}{$\begin{array}{c}\text { Shell } \\
\text { ratio } \\
(\%)\end{array}$} & \multirow{2}{*}{$\begin{array}{l}\text { Pupati } \\
\text { on } \\
\text { rate } \\
(\%)\end{array}$} & \multirow{2}{*}{$\begin{array}{l}\text { Filament } \\
\text { length (m }\end{array}$} \\
\hline & & & & By No. & $\begin{array}{l}\text { By Wt. } \\
\text { (kg) }\end{array}$ & & & & & \\
\hline 1 & CSR-2 & 520 & 94.05 & 9700 & 14.94 & 1.64 & 0.34 & 20.73 & 93.50 & 752 \\
\hline 2 & CSR-6 & 459 & 94.79 & 9373 & 13.09 & 1.56 & 0.31 & 19.91 & 91.00 & 853 \\
\hline 3 & CSR-26 & 518 & 94.77 & 9640 & 14.71 & 1.61 & 0.32 & 20.14 & 93.33 & 852 \\
\hline 4 & CSR-27 & 523 & 94.81 & 9613 & 14.65 & 1.63 & 0.33 & 20.08 & 93.00 & 859 \\
\hline 5 & CSR-50 & 524 & 95.24 & 9680 & 13.94 & 1.54 & 0.32 & 20.46 & 94.50 & 762 \\
\hline 6 & Pam-114 & 521 & 95.12 & 9640 & 14.83 & 1.69 & 0.35 & 20.54 & 93.67 & 860 \\
\hline 7 & Pam-117 & 520 & 95.03 & 9680 & 14.48 & 1.67 & 0.33 & 20.01 & 93.33 & 763 \\
\hline 8 & APS-4 & 519 & 94.68 & 9580 & 13.98 & 1.56 & 0.32 & 20.20 & 93.50 & 778 \\
\hline 9 & APS-5 & 516 & 95.00 & 9620 & 13.62 & 1.52 & 0.31 & 20.12 & 93.50 & 723 \\
\hline 10 & SK-6 & 526 & 94.28 & 9680 & 13.78 & 1.51 & 0.31 & 20.15 & 93.50 & 699 \\
\hline \multirow[t]{3}{*}{11} & SK-7 & 522 & 94.94 & 9640 & 13.84 & 1.59 & 0.32 & 20.18 & 93.00 & 738 \\
\hline & Avg. & 515 & 94.79 & 9622 & 14.17 & 1.59 & 0.32 & 20.23 & 93.26 & 785 \\
\hline & SD & 19 & 0.36 & 90 & 0.59 & 0.06 & 0.01 & 0.24 & 0.85 & 60 \\
\hline
\end{tabular}

Table 7: Evaluation index values of eleven silkworm parental breeds during autumn, 2019

\begin{tabular}{|c|c|c|c|c|c|c|c|c|c|c|c|}
\hline \multirow{2}{*}{ S.No. } & \multirow{2}{*}{$\begin{array}{l}\text { Parental } \\
\text { breeds }\end{array}$} & \multirow{2}{*}{$\begin{array}{c}\text { Fecun } \\
\text { dity } \\
\text { (No.) }\end{array}$} & \multirow{2}{*}{$\begin{array}{c}\text { Hatchi } \\
\text { ng } \\
(\%)\end{array}$} & \multicolumn{2}{|c|}{$\begin{array}{l}\text { Yield / 10, } 000 \\
\text { larvae brushed }\end{array}$} & \multirow{2}{*}{$\begin{array}{l}\text { Single } \\
\text { cocoon } \\
\text { wt. (g) }\end{array}$} & \multirow{2}{*}{$\begin{array}{c}\text { Single } \\
\text { shell } \\
\text { wt. (g) }\end{array}$} & \multirow{2}{*}{$\begin{array}{l}\text { Shell } \\
\text { ratio } \\
(\%)\end{array}$} & \multirow{2}{*}{$\begin{array}{c}\text { Pupatio } \\
\text { n rate } \\
(\%)\end{array}$} & \multirow{2}{*}{$\begin{array}{l}\text { Filame } \\
\text { nt } \\
\text { length } \\
(\mathrm{m})\end{array}$} & \multirow{2}{*}{$\begin{array}{c}\text { Mean } \\
\text { E.I }\end{array}$} \\
\hline & & & & By No. & $\begin{array}{l}\text { By Wt. } \\
\text { (k) }\end{array}$ & & & & & & \\
\hline 1 & CSR-2 & 52.24 & 29.01 & 58.63 & 63.12 & 58.32 & 63.16 & 70.45 & 52.86 & 44.44 & 54.69 \\
\hline 2 & CSR-6 & 20.17 & 49.96 & 22.29 & 31.69 & 44.37 & 41.44 & 36.87 & 23.37 & 61.24 & 36.82 \\
\hline 3 & CSR-26 & 51.58 & 49.49 & 51.95 & 59.15 & 52.50 & 51.10 & 46.32 & 50.89 & 61.11 & 52.68 \\
\hline 4 & CSR-27 & 53.89 & 50.62 & 48.99 & 58.24 & 56.06 & 53.51 & 43.97 & 46.96 & 62.33 & 52.73 \\
\hline 5 & CSR-50 & 54.79 & 62.61 & 56.40 & 46.10 & 41.45 & 45.06 & 59.31 & 64.66 & 46.13 & 52.95 \\
\hline 6 & Pam-114 & 53.21 & 59.33 & 51.95 & 61.19 & 66.36 & 67.99 & 62.60 & 54.83 & 62.52 & 60.00 \\
\hline 7 & Pam-117 & 52.68 & 56.71 & 56.40 & 55.29 & 62.64 & 58.34 & 41.06 & 50.89 & 46.29 & 53.37 \\
\hline 8 & APS-4 & 52.02 & 46.73 & 45.28 & 46.78 & 44.72 & 45.06 & 48.89 & 52.86 & 48.73 & 47.90 \\
\hline 9 & APS-5 & 50.43 & 55.73 & 49.73 & 40.66 & 37.41 & 37.82 & 45.60 & 52.86 & 39.55 & 45.53 \\
\hline 10 & SK-6 & 55.64 & 35.62 & 56.40 & 43.38 & 37.02 & 37.82 & 46.85 & 52.86 & 35.57 & 44.58 \\
\hline 11 & SK-7 & 53.35 & 54.18 & 51.95 & 44.40 & 49.15 & 48.68 & 48.07 & 46.96 & 42.09 & 48.76 \\
\hline
\end{tabular}

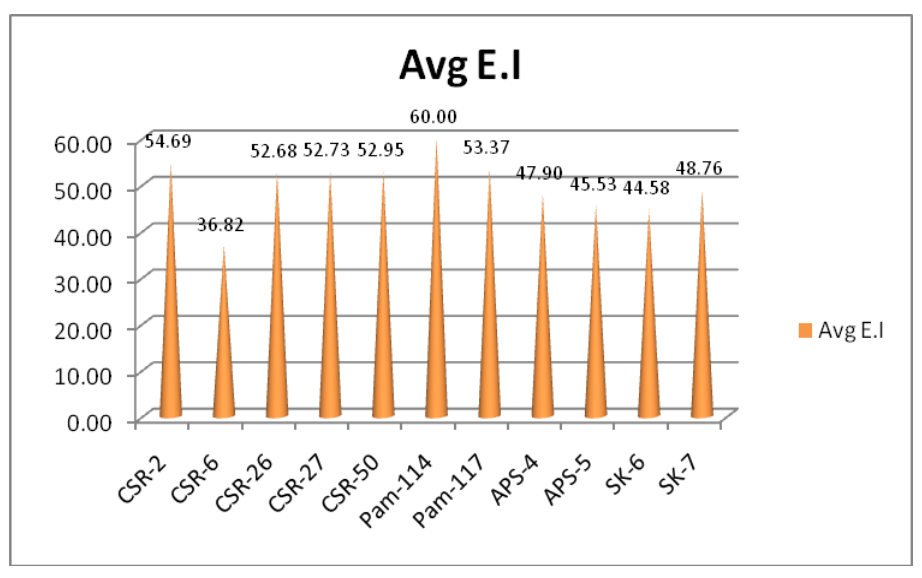

Fig. 3: Average multi-traits evaluation Index values of parental breeds during autumn 2019 


\section{DISCUSSION}

The agro-climatic conditions of the union territory of Jammu and Kashmir enjoy the temperate climate in Kashmir division and sub-tropical in Jammu division. This peculiar climatic conditions specific to the union territory of $J \& K$ is interesting and will not found anywhere else in the country. In Kashmir region, the climatic conditions vary rapidly among three seasons viz., spring, summer and autumn of which spring found to be most favourable for silkworm rearing ${ }^{[18]}$. To find outbreeds suitable for a particular season is the most challenging task for the scientists/researchers in this region. Development of hybrids/ double hybrids suitable for a particular season will only succeed based on the silkworm breed performance in that particular season ${ }^{[19]}$. Silkworm hybrids and double hybrids play an important role in deciding the cocoon production along with silk quality of which silkworm double hybrids are superior over single hybrids ${ }^{[20,21]}$.

In spring 2019, among CSR breeds CSR2, CSR26 and CSR27 performed well compared to CSR6 and CSR50. Rearing results revealed that PAM114, PAM117, APS4 and SK6 recorded El above 50. Interestingly even though spring season found to be favourable for silkworm rearing CSR6, CSR50, APS5 and SK7 recorded EI below 50. In summer 2019, CSR2, CSR26, CSR27, CSR50, PAM114, PAM117 and APS4 recorded EI above 50. A similar trend is observed in autumn season 2019, where CSR2, CSR26, CSR27, CSR50, PAM114 and PAM117 recorded El above 50 excluding CSR6, APS4, APS5, SK6 and SK7. Only CSR6, APS5 and SK7 didn't perform in all the three seasons with $\mathrm{El}$ less than 50 . The present research findings conform to Bharath et al. ${ }^{[22]}$.

Among CSR breeds originated from CSR\&TI, Mysore CSR2, CSR26, CSR27 performed well in all the three seasons except CSR6. CSR50 breed didn't record EI above 50 only in the spring season. Only APS4 breed originated from APSSRDI, Hindupur performed well in spring and summer whereas, SK6 breed originated from CSR\&TI, Berhampore recorded E.I value above 50 during spring only. SK-7 and APS-5 breeds didn't perform well in all the three seasons. PAM114 and PAM117 originated from CSR\&TI, Pampore performed well in all the three seasons. Even though CSR breeds originated from single progenitor the performance of these breeds varies under Kashmir climatic condition. The variation in the performance of the above breeds will be useful to find out the breed to be used for that particular season for the development of season-specific hybrids/ double hybrids ${ }^{[23]}$. Based on the results CSR6, APS5 and SK7 will not be considered for hybrid/ double hybrid production in any of the three season's and APS-4, APS-5 SK-6 and SK-7 specifically not for autumn specific hybrids. The abiotic factors prevailing in summer and autumn season majorly hinders the performance of breeds along with other biotic factors. Hence, the selection of breeds withstanding the unfavourable abiotic and biotic factors will be a key factor for the successful development of hybrids/ double hybrids for Kashmir climatic conditions. Similar studies based on evaluation index values had also been conducted by Nooruldin et al. [24], Rayar [25], Kumaresan et al. ${ }^{[26]}$ and Moorthy et al. ${ }^{[27]}$. The present research findings confirmed by Mir et al. ${ }^{[28]}$ and Bharath et al. ${ }^{[29]}$.

\section{CONCLUSIONS}

The eleven mulberry silkworm parental breeds performed well during spring (CSR2, CSR26, CSR27, PAM114, PAM117, APS4 and SK6), summer (CSR2, CSR26, CSR27, CSR50, PAM114, PAM117 and APS4) and autumn (CSR2, CSR26, CSR27, CSR50, PAM114 and PAM117), 2019. The seasonal variation in the performance of these silkworm breeds during three different seasons (spring, summer and autumn) under Kashmir climatic conditions indicates the degree of genetic variability.

The identified silkworm parental breeds will be utilized for the development of sustainable bivoltine silkworm double hybrids suitable for Kashmir climatic conditions.

\section{ACKNOWLEDGMENTS}

Authors are very thankful to Central Silk Board, Ministry of Textiles, Govt. of India and Central Sericultural Research and Training Institute, Pampore, Jammu and Kashmir (UT) for providing financial assistance and laboratory facilities.

\section{CONTRIBUTION OF AUTHORS}

Research concept- Bharath Kumar Neelaboina, Shivkumar

Research design- Bharath Kumar Neelaboina, Shivkumar Supervision- Materials- Mir Nisar Ahmad

Data collection- Bharath Kumar Neelaboina, Kiran R

Data analysis and interpretation- Bharath Kumar Neelaboina, Mir Nisar Ahmad 
Writing article- Bharath Kumar Neelaboina

Article review- Mir Nisar Ahmad, Shivkumar, Kiran R

Article editing- Mir Nisar Ahmad, S Roy Choudhury

Final approval- Mir Nisar Ahmad, S Roy Choudhury

\section{REFERENCES}

[1] Nagaraju J, Goldsmith MR. Silkworm genomicsProgress and prospects. Curr Sci., 2002; 83(4): 41525.

[2] Bharath KN, Shivkumar, Gani M, Babulal, Ghosh MK. Assessment of performance of autumn crop over spring in temperate region of Jammu \& Kashmir. J Agroecol Natural Resource Manag., 2017; 4(2): 11214.

[3] Kamili AS. New bivoltine silkworm breeds and their hybrids (SKAU-HR-1) Technical Document. Sher-eKashmir University of Agricultural Sciences and Technology of Kashmir (J \& K), 1996.

[4] Trag AR, Kamili AS, Malik GN, Kukiloo FA. Evolution of high yielding bivoltine silkworm, Bombyx mori genotypes. Sericologia., 1992; 32: 321-24.

[5] Sahaf KA, Bhat SA, Mir NA. Sericulture in North-west India with special reference to temperate regionproblems and prospects. National seminar on sericulture development in temperate regionproblems and prospects, 2016; pp. 34-38.

[6] Farooq M, Singh TP, Nooruddin, Rufaie ZH, Baqual M, et al. Second commercial crop to make sericulture a more profitable in Kashmir. Proceedings of regional Seminar on Prospects and Problems of Sericulture as are economic enterprise in North West India, 2006; pp. 275-76.

[7] Malik MA, Kamili AS, Sofi AM, Malik GN, Sabahat A, et al. Second commercial silkworm rearing in Kashmir-A ray of hope. Indian Silk, 2009; 9: 10-11.

[8] Nisar M, Chisti MZ, Khan MA. Studies on the identification of summer specific silkworm Bombyx mori L. hybrids under temperate climatic conditions of Jammu and Kashmir. India. J Int Acad Res Multidisci., 2013; 1(3): 1-14.

[9] Shivkumar, Bharath KN, Mir NA, Shakeel A, Ravindra $M A$, et al. Studies on improvement of quantitative traits of silkworm, Bombyx mori L. during autumn season under temperate climatic conditions of Kashmir. J Entomol Zool Stud., 2018; 6(4): 677-82.

[10]Shivkumar, Bharath KN, Mir NA, Chowdhury SR. Development of Autumn Season Specific Potential and High Yielding Silkworm (Bombyx mori L.) Breeds and Hybrids for Temperate Climate in Jammu and Kashmir. Res J Agril Sci., 2020; 11(1): 62-67.

[11]Basavaraja HK, Nirmal KS, Suresh KN, MalReddy N, Kshama G, et al. New productive bivoltine hybrids. Indian Silk, 1995; 34: 5-9.

[12]Datta RK, Basavaraja HK, MalReddy N, Nirmal KS, Suresh KN, et al. Breeding of new productive bivoltine hybrid, CSR12 x CSR6 of silkworm, Bombyx mori L. Int J Indust Entomol., 2001; 3: 127-33.

[13]Mano Y, Ohyanagi M, Nagayasu K, Murakami A. Breeding of sex-limited larval marking silkworm [Bombyx mori] race, N147 x C145. Bull Natio Inst Seril Entomol Scie., 1991; 2: 1-29.

[14]Bharath KN, Shivkumar, Mir NA, Kiran R, Chowdhury SR. Heterosis Studies on Sustainable Bivoltine Silkworm (Bombyx mori L.) Foundation Crosses Suitable for Kashmir Valley. Res J Agril Sci., 2020; 11(2): 333-40.

[15]Siddiqui AA, Chatterjee SN, Goel AK, Sengupta AK. Genetic divergence in tassar silkworm, Antheraea mylitta D. Sericologia, 1992; 32(3): 425-31.

[16]Bharath KN, Shivkumar, Gulzar AK, Mudasir G, Mir NA, et al. Impact of Climate Change on Agriculture and allied sectors. J Entomol Zool Stud., 2018; 6(5): 426-29.

[17]Mano Y, Nirmalkumar S, Basavaraja HK, MalReddy N, Datta RK. A new method to select promising silkworm breed/hybrid combinations. Indian Silk, 1993; 31(10): 53.

[18]Mir NA, Shivkumar, Bharath KN, Ghosh MK. Evaluation of selected genotypes of bivoltine silkworm Bombyx mori $\mathrm{L}$ during different seasons of Kashmir. J Entomol Zool Stud., 2019; 7(1): 1030-35.

[19]Kiran R, Bharath KN, Shivkumar, Mir NA, Chowdhary $S R$. Scope for region and season specific mulberry silkworm (Bombyx mori L.) in temperate region of Jammu and Kashmir. Res J Agril Sci., 2019; 10(5/6): 809-14.

[20]Bindroo BB, Begum AN, Reddy NM. New Bivoltine Silkworm Double hybrid, JAYACHAMARAJA (CSR50 X CSR52) X (CSR51 X CSR53) for high egg recovery, crop stability and Silk productivity. Technical Bulletin No. 10. CSR \& TI, Mysore, 2014.

[21]Nirmal S, Tara JS, Tayal MK, Avinder K, Kalpana S, et al. Evaluation of different silkworm hybrids in Jammu region. Int J Adv Res., 2016; 4(8), 201-04. 
[22]Bharath KN, Shivkumar, Mir NA, Ghosh MK. Studies on the Performance of Some Silkworm, Bombyx mori $\mathrm{L}$, Breeds in Temperate Region of Jammu and Kashmir, India. Int J Curr Microbiol App Sci., 2018a; 7(11): 2192-01.

[23]Bharath KN, Shivkumar, Mir NA, Ghosh MK. Evaluation of elite bivoltine silkworm (Bombyx mori L.) foundation crosses suitable for temperate region of Jammu \& Kashmir, India. Int J Curr Microbiol App Sci., 2019a; 8(1): 2980-90.

[24]Nooruldin S, Bhat SA, Malik MA, Khan IL, Sahaf KA. Comparative performance of silkworm, Bombyx mori L. hybrids during different seasons under Kashmir climatic conditions. Green Farming, 2014; 6(6): 139295.

[25]Rayar SG. Use of Evaluation Index for Selecting Potential Parents for Silkworm Breeding. Karnataka J Agric Sci., 2007; 20: 420-21.
[26] Kumaresan P, Sinha RK, Sahni NK, Sekar S. Genetic variability and selection indices for economic quantitative traits of multivoltine mulberry silkworm (Bombyx mori L.) genotypes. Sericologia, 2000; 40: 595-05.

[27]Moorthy SM, Das SK, Kar NB, Raje US. Breeding of bivoltine breeds of Bombyx mori L suitable for variable climatic conditions of the tropics. Int J Indust Entomol., 2007; 14: 99- 05.

[28]Mir NA, Bharath KN, Shivkumar, Ghosh MK. Evaluation of Genetic Potential of some Genotypes of Silkworm Bombyx mori L under Temperate Climatic conditions. J Crop Weed, 2018; 14(3): 88-93.

[29]Bharath KN, Shivkumar, Mir NA, Ghosh MK. Evaluation of bivoltine mulberry silkworm Bombyxmori $\mathrm{L}$ breeds suitable for temperate region of Jammu and Kashmir, India, J Entomol Zool Stud., 2019; 7(1): 423-27. 